\title{
A REVIEW: BRAIN TUMOR SEGMENTATION USING K-MEANS CLUSTERING FOR DETECTION AND CALCULATION OF TUMOR
}

\author{
AREA \\ S. P. Mahale ${ }^{1}$, R. K. Kanhe ${ }^{2}$ \\ ${ }^{I}$ PG Research Student, Department of Electronics and Telecommunication Engineering, G. S. Mandal's Maharashtra \\ Institute of Technology, Aurangabad (MS), India \\ ${ }^{2}$ Associate Professor, Department of Electronics and Telecommunication Engineering, G. S. Mandal's Maharashtra \\ Institute of Technology, Aurangabad (MS), India
}

\begin{abstract}
The most challenging and emerging field is medical image processing. For the detection and identification of tumor in MRI of brain is implemented here. The traditional method for brain medical resonance imaging and the detection of tumor is done by human inspection which is operator dependent. Segmentation is done by operator in the clinical environment which is very tedious task and time consuming work. Segmentation of MRI of brain is complicated in this medical imaging field except few presented methods. Brain MRI can be divided into various regions mainly soft tissues like white matter, cerebrospinal fluid, gray matter etc. Using this process of segmentation, the location and size of tumor may be found evaluated. The present methodology proposed here consisting of preprocessing like removal of noise, process of segmentation and some morphological operations which are the basic steps in the image processing tool. Extraction and detection of tumor from brain MRI scan images is done by using k-means clustering method in MATLAB software.
\end{abstract}

Keywords: MRI (Magnetic Resonance Imaging), K-means Clustering.

\section{INTRODUCTION}

Brain tumor is the main cause for increasing the mortality among adults and in children also. By studying few researches, the numbers of people has been found, are becoming victim of brain tumors has increased tremendously.

To improve the image quality and analysis approach from different applications, the image processing tool is used. It helps to improve the accuracy but it is time consuming [1]. Image processing has different application areas like biometric, remote sensing, security and medical. The medical diagnosis purpose is one of the application areas. Current research is on detection of Brain Tumor and its area calculation using image processing. It is an implementation of automated calculation of tumor area [2].

When the growth of tissues becomes uncontrolled in human brain is called tumor and when it converts into a cancer, it becomes life threatening and hence it is very necessary to detect the location and area of tumor means actual size of tumor by using medical imaging. Segmentation of brain MRI plays an important role in locating tumor in magnetic resonance image [3]. For this purpose there are different techniques which are used to capture images such as Computer Tomography (CT), X-Ray and Magnetic Resonance Imaging. But among these methods the MRI is advantages than other because it provides more useful information of anatomical structure which is used for the pathological and clinical studies [4]. The segmentation of medical image is a difficult task due to complexity of the anatomy that is being imaged. Many times it is impossible to detect certain structures without detailed knowledge regarding anatomy. Due to this the process of segmentation becomes a difficult task. Either the information is built in that system or built by an operator. For segmentation process there are many methods which are convenient and used for segmentation purpose such as edge detection, region growing, histogram equalization etc. They need human interaction is the main problem with these methods for reliable segmentation and accurate work. To overcome this problem K-means clustering method is used for segmentation [7].

\section{TRADITIONAL APPROACH}

The traditional method used to detect the tumor from MRI scan images is such a way that radiologist detect and manually calculate size of tumor from CT scan images. 


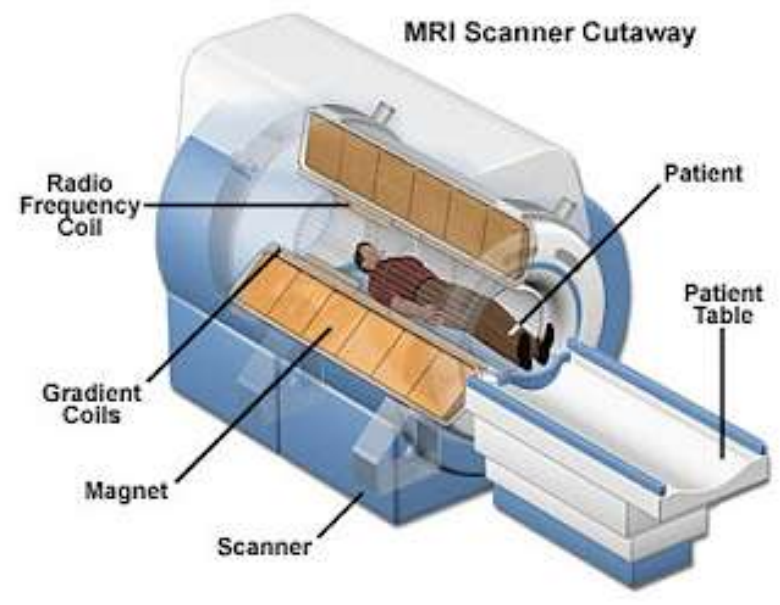

Fig. 1 MRI Scanner

Usually the Computer Tomography technique is used for monitoring purpose of these images of damaged brain parts. The Computer Tomography Scan images are in the form of color image and the detection of tumor from the image becomes easy.

The cerebrum part in the parietal section of the head is displayed in the form of the gray color and the veins, arteries are displayed like creamish white color using CTScan images.

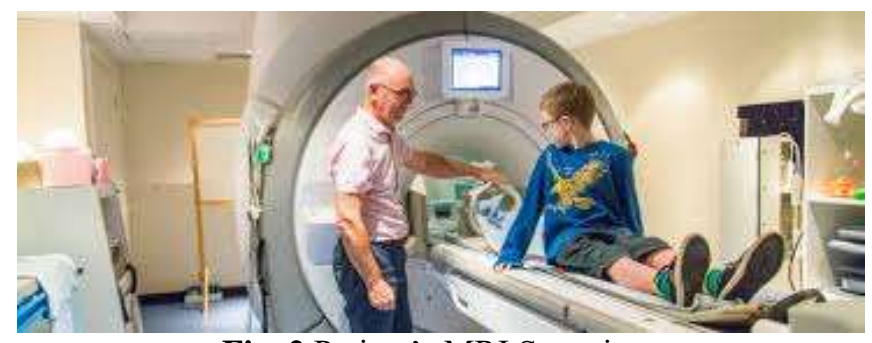

Fig. 2 Patient's MRI Scanning

The normal patient and the patient having the damaged brain are then differentiated by using this methodology. Again based on these image results the tumor can also be detected. Additionally, to determine tumor patient's response this technique has been practiced since long time and it is time consuming.

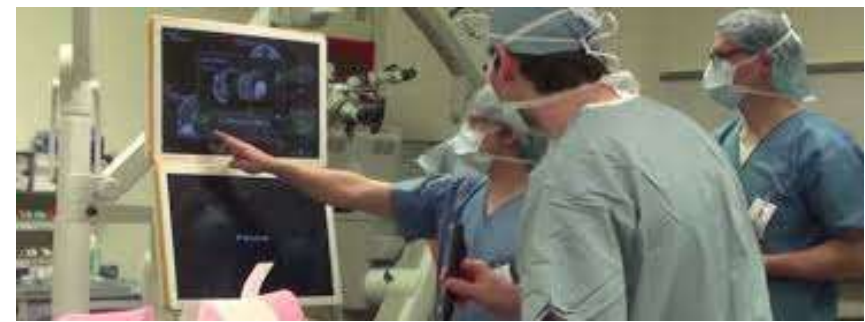

Fig. 3 MRI Monitoring by Doctors

The radiologist has made numbers of measurements of cross-sectional diameter by using Computer Tomography image data. Then, these measurements will be studied by comparing it with previous measurements. Due to some factors, the lesion's measurement of the diameter fails to represent the exact assessment regarding the size of tumor.

\section{Lesion's Irregularity}

These lesions may not adequately represented by diameters, that grow other than sphere shape..

\section{Scanning Levels}

At the same point or spot, the lesions may not be exactly captured.

Hence, it affects the measurement lesions diameter, which causes difficulty in calculation of tumor area.

\section{MODERN APPROACH}

The Modern Approach for Detection and Identification of Tumor from brain MRI consisting of Input Image Selection, Pre-processing of input image, Segmentation, Feature Extraction and Tumor area calculation.

Algorithm's steps:

1) Selection of Image.

2) Pre-processing of data.

3) K-means clustering segmentation.

4) Extraction of features.

5) Tumor Area Calculation.

\subsection{Selection of Input Image}

Patients MRI scan image of brain is taken as an input for the designed system.

\subsection{Pre-Processing of Data}

An input MRI image is converted according to the need of next level is called as preprocessing. The filtering of the noise and other artifacts from that image is carried out and also the sharpening of edges in the image. Also the conversion from RGB to gray and Reshaping of image takes place, if required here. The median filter is used for removal of noise. But the possibilities of noise arrival are less in this modern image scanning technique. Due to thermal effect it may arrive. The aim of this proposed methodology is to detect, identify and segment the tumor part in healthy cells. And this median filter is used for the process of noise removal for the complete system.

\subsection{K-means Clustering Segmentation}

The purpose of k-means clustering is to cluster the given set if data. K-means is one of the simplest partitions methods of clustering. It is one of the learning algorithms which is unsupervised. Clustering the image means grouping out the pixels depends on some characteristics. In K-means clustering the number of clusters $\mathrm{k}$ has to be defining first. The cluster centers $\mathrm{k}$ has to be chosen randomly. Then distance between these cluster centers and pixels are calculated. Every pixel is individually compared with all cluster centers with the help of distance formula. The pixel is moved to particular cluster which has shortest distance among all. This process is continuous until the centre converges. 
Algorithm:

1. Give no. of cluster value $\mathrm{k}$.

2. Choose k cluster centers randomly.

3. Calculate mean.

4. Calculate distance between each pixel to cluster center.

5. If distance is near to center then moved to that cluster.

6. Otherwise move to next cluster.

7. Re-estimate the center

\subsection{Feature Extraction}

While extracting the features the cluster extraction from the output of K-means clustering process. The clusters which are extracted from output of k-means are given to the thresholding process. In this process the binary mask is applied over whole image. Due to this binary masking, the dark pixels become darker and the white pixels become brighter. In threshold coding each coefficient of transform is compared with a threshold. If it is below the threshold value then it is considered as zero and if it is more than the threshold, then it is considered as one. In this Methodology, the coefficients whose magnitudes are above threshold are retained.

\subsection{Tumor Area Calculation}

For calculation of tumor area the extracted tumor image is taken as an input then by calculating number of white pixels and transforming it the tumor area is calculated.

Algorithm used for tumor area calculation.

1. Read the input image.

2. Input image is filtered by using median filter to improve the quality of image and for sharpening purpose.

3. Segmentation of filtered image is carried out for grouping out white pixels.

4. Calculate the no. of white pixels.

5. Converting the pixels into $\mathrm{mm} 2$ by using standard value.

( 1 pixel $=0.26432 \mathrm{~mm} 2)$

6. Display the area a.

\section{CONCLUSION}

In today's world, Medical Imaging has become a very useful task and important work. Application part of this image processing or medical imaging can be found in number of areas which are medical field, remote sensing and the electronics. For diagnosis purpose, as we focus on medical applications, image segmentation process is now widely used. In this proposed methodology, we have discussed a system that can be used to detect and identify tumor part of brain using segmentation of brain MRI. Further we may decide its type by finding area of tumor.

\section{REFERENCES}

[1] Rajesh C. Patil, Dr. A. S. Bhalchandra, "Brain Tumor Extraction from MRI images using MATLAB" International Journal of Electronics, Communication and Soft Computing Science and Engineering ISSN: 2277-9477, Volume 2, Issue 1.
[2] Gauri Anandgaonkar, Dr. Ganesh Sable, "Brain Tumor Detection and Identification from T1 post contrast MR Images using Cluster based Segmentation" International Journal of Science and Research (IJSR), ISSN (Online): 2319-7064.

[3] Kailas Sinha, G. R. Sinha, "Efficient Segmentation Methods for tumor detection in MRI Images" 2014 IEEE Student's Conference on Electrical, Electronics and Computer.

[4] Gauri Anandgaonkar, Dr. Ganesh Sable, "Detection and Identification of Brain Tumor in Brain MR Images using Fuzzy C-Means Segmentation" International Journal of Advanced Research in Computer and Communication Engineering Volume 2, Issue 10, October 2013.

[5] Divya Kaushik, Utkarsha Singh, Paridhi Singhal, Vijai Singh, "Brain Tumor Segmentation using Genetic Algorithm" International Journal of Computer Applications (IJCA) (0975-8887) INTERNATIONAL Conference on Advances in Computer Engineering and Applications (ICACEA) at IMSEC, GZB.

[6] Sneha Khare, Neelesh Gupta, Vibhanshu Srivastava, "Genetic algorithm employed to detect brain tumor in MRI image" International Conference on Cloud, Big data and Trust 2013, Nov 13-15, RGPV.

[7] Prof. B. K. Saptalakar, Miss. Rajeshwari. H. "Segmentation based detection of Brain Tumor" B. K. Saptalakar, et al International Journal of Computer and Electronic Research [Volume 2, Issue 1, February 2013].

[8] Kailas Sinha, G. R. Sinha, "Comparative analysis of optimized k-means and c-means clustering methods of segmentation of Brain MRI Images for Tumor Extraction" Proceedings of international conference on "Emerging research in computing, information, communication and applications" ERCICA 2013 ISBN: 9789351071020.

[9] Shital Agrawal, Dr. S. R. Gupta, "Detection of Brain Tumor using different Edge Detection algorithms" Research article/ April 2014, International Journal of Emerging Research in Management and Technology. 